\title{
A little help from steroids in oncology
}

\author{
Dominique Lossignol \\ Supportive and Palliative Care Unit, Institut Jules Bordet-Université Libre de Bruxelles, 1000 Brussels, Belgium
}

\section{ABSTRACT}

Steroids are widely used in oncology and have been demonstrated to possess an anticancer effect or antiswelling effect. They are considered to improve refractory symptoms such as dyspnea or gastrointestinal (GI) obstruction. However, their roles in nonspecific indications are not well proved. Clinical practice and several studies suggest that corticosteroids may be effective in the treatment of bone and neuropathic pain, when administered along with opioids and with other adjuvant analgesics. The decrease in pain intensity is probably connected with both anti-inflammatory and antiswelling effects as well as modulation of neuroimmune interactions and an inhibition of angiogenesis.

Key words: steroids, cancer, pain, indication

\section{INTRODUCTION}

Steroids are widely used in oncology, from cancer curative treatment to supportive care. They have a demonstrated anticancer effect (i.e., leukemia or lymphoma management) or antiswelling effect (i.e., brain tumor or brain metastases) and are considered to improve refractory symptoms such as dyspnea or gastrointestinal (GI) obstruction. Although this could contribute to the assertion that they are always useful, there is little evidence for the effectiveness and toxicity of corticosteroids in cancer. Some uncontrolled studies reported benefits of corticosteroids on pain and symptom control, but questions remain:

Dr. Dominique Lossignol, Supportive Dr. Domiliue Lossignol, Suppotive Bordet-Université Libre de Bruxelles, 1000 Brussels, Belgium

Email: dominique.lossignol@bordet.be

\begin{tabular}{|c|}
\hline Access this article online \\
\hline $\begin{array}{l}\text { Website: } \\
\text { www.intern-med.com }\end{array}$ \\
\hline $\begin{array}{l}\text { DOI: } \\
\text { 10.1515/jtim-2016-0011 }\end{array}$ \\
\hline Quick Response Code: \\
\hline 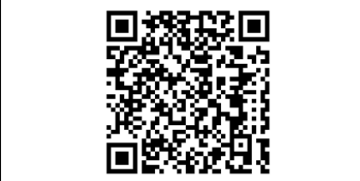 \\
\hline
\end{tabular}

- Is pain an indication for the use of steroids?

- What are the indications?

- How long are steroids effective in symptom management, including pain?

- What is the most effective steroid dose?

- What are the most common adverse effects in cancer patients?

- Are steroids “overused" in palliative care?

\section{PAIN}

Pain is a major problem in cancer as it occurs in $30-50 \%$ patients in earlier stages and in $70-90 \%$ of these patients with advanced disease. The three-step framework for cancer-related pain management was published for the first time in 1986. Step I recommends nonopioid analgesics (nonsteroidal anti-inflammatory drugs [NSAIDs], paracetamol) for mild pain. Step II specifies the use of weak opioids for moderate pain (codeine, tramadol). Step III comprises the use of strong opioids for severe pain (morphine, methadone, fentanyl, hydromorphone).

To achieve better pain relief, an appropriate application of adjuvant analgesics (i.e., for neuropathic pain), supportive drugs (for the prevention and treatment of opioid adverse effects), and nonpharmacological measures such as radiotherapy and invasive procedures (nerve blockades and neurolytic blocks) should be considered and applied. In patients with bone pain, opioids may be combined with NSAIDs and bisphosphonates along with local or systemic radiotherapy. The superiority of steroids over NSAIDs has not been demonstrated and side effects are of concern. Only case reports have demonstrated the effectiveness of corticosteroids in bone pain treatment. Given the present knowledge, a weak recommendation for the use of corticosteroids in cancer patients with pain is found. The evidence supports that a moderate dose of corticosteroids, such as 
methylprednisolone $32 \mathrm{mg}$, may contribute to analgesia and seems to be well tolerated. The corticosteroid medication should be discontinued after a week if there is no pain relief. ${ }^{[1]}$ There is at least one complete review on the role on steroids in cancer-related pain management. ${ }^{[2]}$ The conclusions are quite clear: "Clinical practice and several studies suggest that corticosteroids may be effective in the treatment of bone and neuropathic pain, when administered along with opioids and with other adjuvant analgesics. The decrease in pain intensity is probably connected with both the anti-inflammatory and anti-swelling effects as well as modulation of neuroimmune interactions. It seems that dexamethasone may be commonly used for cancer pain management due to its high potency, long duration of action and minimal mineralocorticoid effect. A proposed starting daily dose is $8 \mathrm{mg}$ (orally, subcutaneously, or intravenously) with subsequent adjustment to the analgesia achieved and adverse effects. It is estimated that positive effect of steroids lasted from 3 to 6 weeks."

The analgesic properties of corticosteroids should be confirmed in a randomized trial.

Accordingly, only a qualitative analysis could be made.

\section{SUPPORTIVE CARE}

The main indications included treatment for raised intracranial pressure and to give a "boost." The most common specific indications for treatment with dexamethasone were spinal cord compression, cerebral metastases, lymphangitis carcinomatosa, and bowel obstruction. The most common nonspecific indications comprised anorexia, nausea, low mood, pain, and vomiting.

In an uncontrolled study among 106 consecutive patients with advanced cancer dexamethasone treatment was started according to the established prescription policy. ${ }^{[3]}$ The most common specific indications for treatment with dexamethasone were spinal cord compression, cerebral metastases, lymphangitic carcinomatosis, and bowel obstruction. The most common nonspecific indications comprised anorexia, nausea, low mood, pain, and vomiting. The median duration of steroid use was 21.5 days (range: 1-89 days). Symptom scores improved during the therapy compared with the baseline in most patients for anorexia, nausea, pain, low mood, vomiting, and weakness, but not with respect to dyspnea and poor mobility. The foremost reason for discontinuation of steroids is loss of the oral route. There is no consensus regarding a systematic shift to IV route.

\section{GI TRACT OCCLUSION}

This situation is complex in terms of treatment and management. The recommended strategy involves a clinical and radiological evaluation, of which computed tomography (CT) of the abdomen is a crucial component. ${ }^{[4]}$ The results, together with an analysis of the prognostic criteria, are used to determine whether surgery or stenting is the best option. In most patients neither option is feasible and the main emphasis is on the role and administration of various symptomatic medications such as glucocorticoids, antiemetic agents, analgesics, and antisecretory agents (anticholinergic drugs, somatostatin analogues, and proton-pump inhibitors). Nasogastric tube feeding is no longer used routinely and should instead be discussed on a case-by-case basis. Recent studies have confirmed the efficacy of somatostatin analogues in relieving obstruction-related symptoms such as nausea, vomiting, and pain. Although steroids are not the drug of choice, they are part of the treatment and should not be avoided.

\section{APPETITE}

Moertel et al. ${ }^{[5]}$ reported a significant improvement in appetite in a randomized, double-blind, placebo-controlled study of dexamethasone in 116 patients with advanced GI cancer after 4 weeks, although their weight did not increase. Improvements in depression, appetite, and food consumption also were observed. Strength was insignificantly improved, and performance status and survival were not different from control.

Corticosteroids are beneficial in treating anorexia in palliative care patients with malignancies; however, there is no evidence for their use in anorexia due to end-stage nonmalignant disease. There is insufficient evidence to recommend any particular corticosteroid drug over another, or to recommend a dosing regimen. ${ }^{[6]}$

\section{DYSPNEA}

Steroids are proposed in the case of pulmonary lymphangitis. The effect on dyspnea is short in duration because of the progressive nature of the disease. Intercostal muscle atrophy is of concern, especially in debilitated or cachectic patients. Various schedules have been proposed but no one has been compared to another.

\section{FATIGUE}

Recent studies confirm the short-term benefit of corticosteroids for the symptomatic treatment of cancer-related fatigue and anorexia cachexia in advanced incurable cancer. Long-term use of corticosteroids is not recommended at this time because of the risk of debilitating side effects and no evidence of its long-term benefits. Future studies are needed to determine the optimal dose, type, and the role of corticosteroid rotation so as to optimize long-term efficacy and minimize side effects. ${ }^{[7]}$

\section{NEURO-ONCOLOGY}

Glucocorticoids are powerful agents that have been widely used for decades by neuro-oncologists in the management 
of brain tumor patients. ${ }^{[8]}$ Despite their long history in medicine, the exact mechanisms by which glucocorticoids exert their anti-inflammatory and antiedema actions are not completely understood. Moreover, glucocorticoids may directly influence the properties and behavior of brain tumor cells and normal neural progenitors, carrying the risk of interference with the success of cancer treatment. Glucocorticoids are associated with numerous and potentially serious side effects, especially in patients treated over long time periods. Several novel molecular targeted agents, such as bevacizumab, designed to inhibit pathways involved in angiogenesis, have shown to reduce braintumor-associated cerebral edema, allowing the reduction of glucocorticoid use in brain tumor patients.

\section{WHICH STEROID, WHICH DOSE(S)?}

Hanks et al. ${ }^{[9]}$ reported an uncontrolled study examining the effects of prednisolone or dexamethasone in 218 (58\%) out of a total of 373 patients with advanced cancer admitted to the inpatient unit. Patients received either prednisolone $(n=121)$ or dexamethasone $(n=95)$ in starting doses of $10-30 \mathrm{mg}$ or 4-16 $\mathrm{mg}$ daily, respectively. The maintenance daily doses varied considerably from 5 to $20 \mathrm{mg}$ of prednisolone and from $0.5 \mathrm{mg}$ on alternate days to $4 \mathrm{mg}$ twice daily of dexamethasone. There was no difference in response rates between the two drugs.

The problem of appropriate steroid dose and its efficacy is raised in the literature and clinical practice. In a randomized double-blind trial, Vecht et al. ${ }^{[10]}$ compared initial dexamethasone dose of $10 \mathrm{mg}$ versus $100 \mathrm{mg}$ in 37 patients with spinal cord compression who then received radiotherapy. No significant differences were found between the patient groups with respect to pain relief.

\section{CONCLUSIONS}

Corticosteroids have an established role in specific indications in cancer patients such as spinal cord compression, superior vena cava syndrome, brain metastases with raised intracranial pressure, and bowel obstruction. Their role in nonspecific indications is not well proved. Clinical practice and several studies suggest that corticosteroids may be effective in the treatment of bone and neuropathic pain, when administered along with opioids and with other adjuvant analgesics. The decrease in pain intensity is probably connected with both the antiinflammatory and antiswelling effects as well as modulation of neuroimmune interactions. Another mechanism of action could be an inhibition of angiogenesis.

It seems that dexamethasone may be commonly used for cancer pain management due to its high potency, long duration of action, and minimal mineralocorticoid effect. A proposed starting daily dose is $8 \mathrm{mg}$ (orally, subcutaneously, or intravenously) with subsequent adjustment to the analgesia achieved and adverse effects. The lowest effective dose should be used and patients must be followed up regularly to assess benefits and risks associated with the therapy. In the situation when the general condition of a patient deteriorates and the patient is no longer able to swallow medications that are taken orally, it is rather recommended not to stop the treatment abruptly but to continue dexamethasone administration by the subcutaneous route.

Patients with severe pain intensity of bone and neuropathic origin should also be considered for the use of analgesic ladder step 3 opioids (opioids for moderate to severe pain, strong opioids) without moving up the analgesic ladder. Other co-analgesics appropriate for bone and neuropathic pain should also be considered. Future studies that would take patients' quality of life into consideration and could establish the role of corticosteroids in pain and other symptom treatment are urgently needed.

\section{Conflicts of Interest}

None declared.

\section{REFERENCES}

1. Paulsen $\varnothing$, Aass N, Kaasa S, Dale O. Do Corticosteroids Provide Analgesic Effects in Cancer Patients? A Systematic Literature Review. J Pain Symp Manage 2013;46:96-105.

2. Leppert W, Buss Y. The role of corticosteroids in the treatment of pain in cancer patients. Curr Pain Headach Rep 2012; 16: 307-13.

3. Hardy JR, Rees E, Ling J, Burman R, Feuer D, Broadley K, et al. A prospective survey of the use of dexamethasone on a palliative care unit. Palliat Med 2001; 15:3-8.

4. Laval G, Marcelin-Benazech B, Guirimand, Chauvenet L, Copel L,Durand A, F et al. Recommendations for bowel obstruction with peritoneal carcinomatosis. J Pain Symptom Manage 2014 75-91.

5. Moertel CG, Schutt AJ, Reitemei RJ, Hahn RG. Corticosteroid therapy of preterminal gastrointestinal cancer. Cancer 1974; 33:1607-9.

6. Miller S, McNutt L, McCann MA, McCorry N. Use of Corticosteroids for Anorexia in Palliative Medicine: A Systematic Review. J Palliat Med 2014;17:482-5.

7. Yennurajalingam S, Bruera E. Role of corticosteroids for fatigue in advanced incurable cancer: is it a 'wonder drug' or 'deal with the devil'. Curr Opin Support Palliat Care 2014;8:346-51.

8. Dietrich J, Rao K, Pastorino S, Kesari S. Corticosteroids in brain cancer patients: benefits and pitfalls. Expert Rev Clin Pharmacol 2011;4:233-42.

9. Hanks GW, Trueman T, Twycross RG. Corticosteroids in terminal cancer-a prospective analysis of current practice. Postgrad Med J 1983; 59:702-6.

10. Vecht CJ, Haaxmareiche H, Vanputten WLJ, Devisser M, Vries EP, Twin metastatic spinal-cord compression. Neurology 1989; 39:1255-7.

How to cite this article: Lossignol D. A little help from steroids in oncology. J Transl Intern Med 2016; 4: 52-4. 